\title{
GABAergic Excitation of Spider Mechanoreceptors Increases Information Capacity by Increasing Entropy Rather than Decreasing Jitter
}

\author{
Keram Pfeiffer and Andrew S. French \\ Department of Physiology and Biophysics, Dalhousie University, Halifax, Nova Scotia B3H 1X5, Canada
}

\begin{abstract}
Neurotransmitter chemicals excite or inhibit a range of sensory afferents and sensory pathways. These changes in firing rate or static sensitivity can also be associated with changes in dynamic sensitivity or membrane noise and thus action potential timing. We measured action potential firing produced by random mechanical stimulation of spider mechanoreceptor neurons during long-duration excitation by the $\mathrm{GABA}_{\mathrm{A}}$ agonist muscimol. Information capacity was estimated from signal-to-noise ratio by averaging responses to repeated identical stimulation sequences. Information capacity was also estimated from the coherence function between input and output signals. Entropy rate was estimated by a data compression algorithm and maximum entropy rate from the firing rate. Action potential timing variability, or jitter, was measured as normalized interspike interval distance. Muscimol increased firing rate, information capacity, and entropy rate, but jitter was unchanged. We compared these data with the effects of increasing firing rate by current injection. Our results indicate that the major increase in information capacity by neurotransmitter action arose from the increased entropy rate produced by increased firing rate, not from reduction in membrane noise and action potential jitter.
\end{abstract}

\section{Introduction}

Neuromodulation commonly refers to processes in which chemical compounds, released locally or diffusely, modulate the responsiveness of neurons that are transmitting signals received from stronger or more direct inputs. Neuromodulation can increase or decrease excitability, as seen by changes in membrane potential and action potential firing rates (Matheson, 1997; Page and Blackshaw, 1999; Rudomin and Schmidt, 1999; Torkkeli and Panek, 2002; Birmingham et al., 2003; Widmer et al., 2005). It can also affect dynamic properties of neuronal responses or spontaneous firing patterns (Ramirez et al., 1993; Matheson, 1997; Birmingham et al., 2003; Pfeiffer et al., 2009).

Neuromodulation has also been reported to change the reliability of action potential timing (or its inverse, jitter) and thus the amount of information that can be transmitted by the neuron (Billimoria et al., 2006). Action potential timing jitter is a well known neuronal phenomenon with several possible sources (Rieke et al., 1997; Billimoria et al., 2006; Rokem et al., 2006; Kreuz et al., 2007). Reducing jitter can increase the fidelity of information transmission (Aldworth et al., 2005).

Here, we ask whether excitability or action potential timing reliability dominates changes in information capacity during neuromodulation of a single mechanosensory neuron. Mechanoreceptor afferents entering the CNSs of vertebrates and invertebrates prob-

Received June 11, 2009; revised July 18, 2009; accepted July 22, 2009

This work was supported by the Canadian Institutes of Health Research and the Nova Scotia Health Research Foundation. Shannon Meisner provided excellent technical assistance.

Correspondence should be addressed Andrew S. French, Department of Physiology and Biophysics, Dalhousie University, Halifax, NS B3H 1X5, Canada. E-mail: andrew.french@dal.ca.

D0I:10.1523/JNEUROSCI.2744-09.2009

Copyright $\odot 2009$ Society for Neuroscience $\quad$ 0270-6474/09/2910989-06\$15.00/0 ably all receive presynaptic neuromodulation (Rudomin and Schmidt, 1999; Torkkeli and Panek, 2002), but mechanoreceptors and pain receptors can also be modulated in the periphery, by either direct efferent innervation or circulating neuromodulators. Peripheral modulation is well known in invertebrates (Torkkeli and Panek, 2002) and probably widespread in vertebrates, as shown by the sensitivity of some vertebrate mechanoreceptors to sympathetic activity (Loewenstein, 1956), GABAergic inhibition of vagal mechanoreceptors (Page and Blackshaw, 1999), and the presence of glutamate receptors on nociceptive terminals (Carlton, 2001).

We showed previously that information capacity in spider mechanoreceptor neurons rises as the firing rate is increased by current injection (French et al., 2001), octopamine (Widmer et al., 2005), or $\mathrm{GABA}_{\mathrm{A}}$ agonists (Pfeiffer et al., 2009). Here, we asked whether this increased information capacity is caused by decreased jitter or by other mechanisms. Our data indicate that increased firing rate increases the maximum entropy rate, which is then used for sensory encoding, and increases the information capacity. In contrast, decreased jitter plays a relatively minor role.

\section{Materials and Methods}

Animal preparation and electrophysiology. Spiders, Cupiennius salei, were maintained in a laboratory colony at room temperature $\left(22 \pm 2^{\circ} \mathrm{C}\right)$ and a 13/11 h light/dark cycle. For all experiments, legs from adult spiders were autotomized following a protocol approved by the Dalhousie University Committee on Laboratory Animals. Patella cuticle containing the intact VS-3 slit-sense organ was cut from the leg and waxed to a Plexiglas holder that permitted access to both the outer and inner surfaces of the organ (Fig. 1) (Juusola et al., 1994; Seyfarth and French, 1994).

Neurons were visualized using an Axioskop 2 FS Plus upright compound microscope with an Achroplan $40 \times$ water-immersion objective 
(Carl Zeiss), mounted on a gas-driven vibration isolation table inside a Faraday cage (Technical Manufacturing). Sharp borosilicate glass microelectrodes (outer diameter, $1 \mathrm{~mm}$; inner diameter, $0.5 \mathrm{~mm}$; Hilgenberg) were pulled using a P-2000 horizontal laser puller (Sutter Instrument). Electrodes were filled with $2.5 \mathrm{M} \mathrm{KCl}$ and had resistances between 40 and $100 \mathrm{M} \Omega$ in solution. Recordings were made in discontinuous single-electrode currentclamp or voltage-clamp controlled currentclamp (VCcCC) mode (Sutor et al., 2003) using a SEC-10LX amplifier with a VCcCC addendum (NPI Electronic). Switching frequencies between 18 and $20 \mathrm{kHz}$ and a duty cycle of $1 / 4$ (current passing/voltage recording) were used. The voltage was low-pass filtered at $33.3 \mathrm{kHz}$, and the current signal was filtered at $3.3 \mathrm{kHz}$ by the amplifier. Neuronal somata were impaled with the microelectrodes using a PatchStar micromanipulator (Scientifica).

Neurons were stimulated mechanically using a P-841.10 piezoelectric stimulator driven by a E-505.00 LVPZT amplifier (Physik Instrumente) that pushed a glass probe against the slits from below (Fig. 1). Deflection of $0.3 \mu \mathrm{m}$ was adequate to evoke action potentials. The mechanical stimulator response was low pass, with a corner frequency of $70 \mathrm{~Hz}$. IBM-compatible personal computers were used for all data recording and stimulation using customwritten software. Current, voltage, and mechanical signals were provided by the computer via a 12-bit digital-to-analog converter and recorded via a 16-bit analog-to-digital converter (National Instruments).

Chemicals and drug application. All chemicals were purchased from Sigma. The preparation was continuously superfused with spider saline (in mm: $223 \mathrm{NaCl}, 6.8 \mathrm{KCl}, 8 \mathrm{CaCl}_{2}, 5.1$ $\mathrm{MgCl}_{2}, 10$ HEPES, and 17 glucose, $\mathrm{pH} 7.8$ ) via plastic tubing with a flow rate of $0.5-1 \mathrm{ml} / \mathrm{min}$ (Fig. 1). The superfusion solution was injected at the side of the $40 \times$ objective. The total volume of saline in the recording chamber was $\sim 3$ $\mathrm{ml}$. Receptor agonists were aliquoted and kept frozen until just before each experiment. Muscimol was initially dissolved in $0.05 \mathrm{M} \mathrm{HCl}$.

Stimulation. The stimulus signal consisted of pseudorandom Gaussian white noise generated by the computer via a 33-bit binary sequence algorithm. To obtain averaged responses, repeated identical segments of the random stimulus were concatenated to produce a continuous signal that drove the mechanical stimulator. The random signal had a time resolution of $0.1 \mathrm{~ms}$, and repeat segments were $5.12 \mathrm{~s}$ duration. Total stimulation time for each experiment was at least $300 \mathrm{~s}$, giving $\sim 60$ individual repeats of the random stimulus. Intracellular action potential signals were also sampled at $0.1 \mathrm{~ms}$ resolution.

Measuring information capacity by averaging. The general approach followed procedures used before for analog responses from VS-3 neurons (Juusola and French, 1997). It has also been called the upper bound method (Borst and Theunissen, 1999). Action potentials were detected by a threshold-detection algorithm
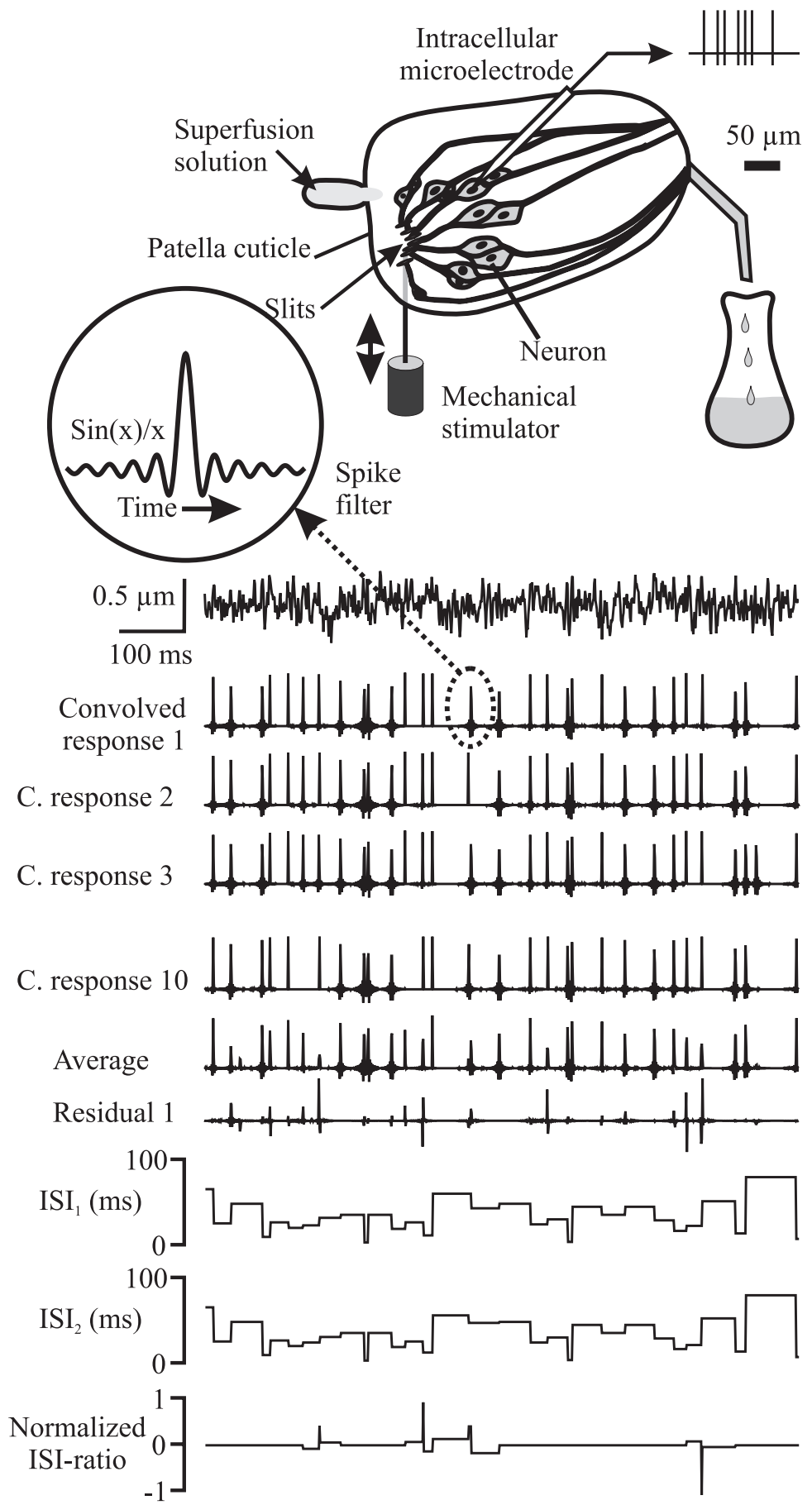

Figure 1. Experimental arrangement and data analysis procedures for measuring the effects of neuromodulation on information transmission by spider VS-3 neurons. Top, Neurons of a VS-3 slit-sense organ exposed in a piece of patella cuticle. A piezo-electric mechanical stimulator caused action potential firing in a neuron that was detected by an intracellular microelectrode. The preparation was continuously superfused with spider saline. Release of muscimol solution $(0.5 \mathrm{ml}$ of $100 \mu \mathrm{m}$ ) close to the neurons took 5-10 s. Traces show an example of repeated stimulation by a randomly varying mechanical signal (top trace). Convolved response traces show action potentials digitally filtered by a $\sin (x) / x$ function (circled inset) to give continuous responses. Note that the sampled amplitudes of the filtered spikes vary depending on spike timing relative to the regular sample points and that spikes close together cause the $\sin (x) / x$ functions to add and subtract their negative and positive excursions (French and Holden, 1971). Convolved responses to 10 repeated random stimulus presentations were averaged (convolved responses $1,2,3, \ldots 10$ are shown here), and then the average was subtracted from each response to give 10 residuals. Signal-to-noise ratios from the average and residual values were used to estimate information capacity. The same responses were also converted to the frequency domain to estimate the coherence function and thus another estimate of information capacity. The bottom three traces illustrate calculation of the ISIs and normalized ISI ratio from the same action potential signals (see Materials and Methods). 
(French et al., 2001) and stored as time of occurrence. They were then digitally filtered by convolution with a $\sin (x) / x$ function and resampled at $1 \mathrm{~ms}$ intervals, to obtain an analog signal band limited to a frequency range of $0-500 \mathrm{~Hz}$. The advantage of using the $\sin (x) / x$ function is that it provides a rectangular sampling window in the frequency domain, removing all frequency components above the Nyquist sampling frequency, without affecting lower frequencies, which is important for accurate estimation of the frequency response function or coherence function (French and Holden, 1971). Sets of 10 responses to the same random input were then summed to obtain an average response, which was subtracted from each of the 10 responses separately, giving 10 residual signals. Figure 1 illustrates this process by showing a section of the repeated random stimulus, a series of responses, the average of 10 responses, and one of the residual segments.

The average response was considered to be the signal resulting from the input stimulus, whereas the residual responses were considered to be the inherent variability, or noise, in the neuron, leading to a signal-tonoise ratio, $\operatorname{SNR}(t)$. Average and residual records were converted to the frequency domain by the fast Fourier transform (FFT) (Cooley and Tukey, 1965). The data were broken into adjacent 512 points segments, and the FFT was applied to each segment in turn. The resulting signal and noise values as functions of frequency were divided to give the SNR and the information capacity (Shannon and Weaver, 1949):

$$
R_{\mathrm{AV}}=\int \log _{2}(1+S N R(f)) d f .
$$

Information capacity as a function of time during the recording was obtained by repeating the above procedure on sets of 10 segments, incrementing the initial segment in time. Mean firing rate during each set of 10 was obtained by counting all action potentials in the set. Firing rate and information capacity as functions of time are shown in the top two traces of Figure 2. This procedure gave a compromise between temporal resolution and variability of the individual estimates. The midpoint of each set of 10 segments was used as the time of measurement.

Measuring information capacity by coherence. The coherence function, $\gamma^{2}(f)$, for each of the sets of 10 segments of data was obtained from the spectra of the input (random) signal, $S_{x x}(f)$, output (digitally filtered action potentials) signal, $S_{y y}(f)$, and the cross-spectrum, $S_{x y}(f)$ (Bendat and Piersol, 1980):

$$
\gamma^{2}(f)=\left\langle S_{x y}(f)^{2}\right\rangle /\left(\left\langle S_{x x}(f)\right\rangle\left\langle S_{y y}(f)\right\rangle\right) .
$$

Spectra were obtained by fast Fourier transform, using 512 point segments, as above. Information capacity was estimated from the following (Juusola and French, 1997):

$$
R_{\mathrm{CO}}=\int \log _{2}\left(1 /\left(1-\gamma^{2}(f)\right)\right) d f,
$$

for the same sets of 10 segments used for the averaging method and plotted against the same time axis (Fig. 2, third trace).

Entropy rate of the action potential signal. Entropy rate, $E$, in bits per second, was estimated by the context-free data compression technique (French et al., 2003). The action potentials, in 1-ms-wide histogram bins, were considered to be a message containing two symbols, 1 and 0 , where 1 indicated the occurrence of an action potential and 0 the absence of an action potential within that sample interval. Repeated substitution was then applied to compress the signal by replacement of repeated symbolic patterns with additional symbols, until the minimum combination of message length and number of symbols was achieved. Entropy was then estimated from the number of bits required to encode each symbol and reconstruct the entire original message without error, divided by the total message length (Jiménez-Montaño et al., 2000; French et al., 2003).

Maximum entropy rate was estimated from:

$$
E_{\max } \approx F \log _{2}(e / F \Delta t),
$$

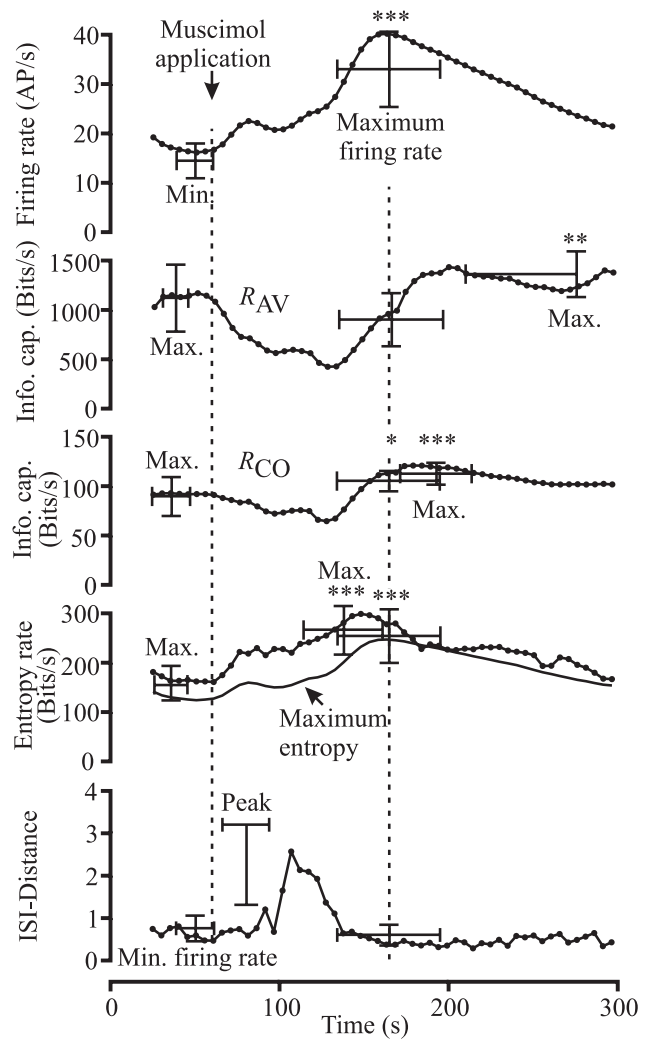

Figure 2. Effects of muscimol on information transmission by VS-3 neurons. Data from one neuron are shown as illustration, with mean $\pm S D$ values from 10 different neurons superimposed. Vertical dashed lines indicate the time of muscimol application $(60 \mathrm{~s})$ and mean time of peak firing $(164.9 \mathrm{~s})$. Muscimol application took $5-10 \mathrm{~s}$. Mean values are shown for the minimum and maximum firing rates, the maximum values of parameters before and after muscimol application, and the maximum values reached throughout the experiments for some parameters. In each case, the SDs of the measurement times are also shown as horizontal bars. Data values are shown in Table $1 . R_{\mathrm{AV}}$, Information capacity by averaging; $R_{\mathrm{CO}}$, information capacity by coherence function.

where $F$ is the firing rate in action potentials per second $(\mathrm{AP} / \mathrm{s})$, and $\Delta t$ is the sample interval (1 ms) (Rieke et al., 1997). Entropy rate and maximum entropy rate were calculated for each set of 10 segments, as above, and plotted against the same time axis (Fig. 2, fourth traces).

Spike train synchrony or jitter. The reliability of action potential firing during repeated presentations of the same input stimulus was estimated from the interspike interval distance, $D(t)$ (Kreuz et al., 2007). The times of action potential occurrence were first used to create interspike interval (ISI) traces for each data segment. Figure 1 illustrates ISI traces for the first two traces of the same recording used for the averaging process, ISI $_{1}$ and $\mathrm{ISI}_{2}$. Then, the ISI of each $5.12 \mathrm{~s}$ segment was compared with the previous segment by constructing a normalized ISI ratio, $I(t)$ :

$$
\begin{aligned}
& I(t)=\mathrm{ISI}_{n} / \mathrm{ISI}_{n-1}-1, \mathrm{ISI}_{n} \leq \mathrm{ISI}_{n-1}, \\
& I(t)=1-\mathrm{ISI}_{n-1} / \mathrm{ISI}_{n}, \mathrm{ISI}_{n}>\mathrm{ISI}_{n-1} .
\end{aligned}
$$

Parameter $I(t)$ is shown in the bottom trace of Figure 1. Finally, the absolute ISI distance parameter, $D$, was calculated from the following (Kreuz et al., 2007):

$$
D=\int I(t) d t .
$$

$D$ was measured for each data segment and plotted against the same time axis (Fig. 2, fifth trace).

ISI distance was chosen as a relatively simple method for comparing reliability between two spike trains that has been tested against other 
Table 1. Effects of muscimol on firing rate, information capacity, entropy, and interspike interval distance in 10 different VS-3 neurons

\begin{tabular}{|c|c|c|c|c|}
\hline \multirow[b]{2}{*}{ Parameter } & \multicolumn{2}{|l|}{ Parameter value } & \multicolumn{2}{|c|}{ Measurement time (s) } \\
\hline & Mean \pm SD & $p$ & Mean \pm SD & $p$ \\
\hline $\begin{array}{l}\text { Firing rate before M } \\
\text { application }\end{array}$ & $14.65 \pm 3.78$ & & 60 & \\
\hline $\begin{array}{l}\text { Lowest firing rate } \\
\text { before M (AP/s) }\end{array}$ & $14.48 \pm 3.57$ & $<0.0001$ & $50.18 \pm 10.74$ & $<0.0001$ \\
\hline $\begin{array}{l}\text { Peak firing rate after } \\
M(A P / s)\end{array}$ & $33.06 \pm 7.62$ & & $164.9 \pm 30.42$ & \\
\hline $\begin{array}{l}\text { Peak } R_{\mathrm{AV}} \text { before } M \\
\text { (bits/s) }\end{array}$ & $1112 \pm 337$ & & $38.4 \pm 7.34$ & \\
\hline $\begin{array}{l}\text { Peak } R_{\mathrm{AV}} \text { after } \mathrm{M} \\
\text { (bits/s) }\end{array}$ & $1354 \pm 230$ & 0.0054 & $272.9 \pm 64.7$ & 0.0054 \\
\hline $\begin{array}{l}R_{\mathrm{AV}} \text { at peak firing } \\
\quad(\mathrm{bits} / \mathrm{s})\end{array}$ & $893.0 \pm 267.0$ & 0.082 & {$[164.9]$} & \\
\hline $\begin{array}{l}\text { Peak } R_{\mathrm{co}} \text { before } M \\
\quad(\mathrm{bit} / \mathrm{s})\end{array}$ & $89.67 \pm 19.72$ & & $35.98 \pm 11.30$ & \\
\hline $\begin{array}{l}\text { Peak } R_{\text {co }} \text { after } M \\
\text { (bits } / s)\end{array}$ & $112.8 \pm 11.09$ & 0.0008 & $192.58 \pm 21.13$ & 0.0155 \\
\hline $\begin{array}{l}R_{C 0} \text { at peak firing } \\
\quad(\mathrm{bits} / \mathrm{s})\end{array}$ & $105.2 \pm 10.24$ & 0.0155 & {$[164.9]$} & \\
\hline $\begin{array}{l}\text { Peak entropy before } \\
\qquad M(b i t s / s)\end{array}$ & $155.6 \pm 31.28$ & & $35.84 \pm 9.65$ & \\
\hline $\begin{array}{l}\text { Peak entropy after } \\
\text { M (bits/s) }\end{array}$ & $266.9 \pm 49.38$ & $<0.0001$ & $137.7 \pm 23.33$ & $0.0054(W)$ \\
\hline $\begin{array}{l}\text { Entropy at peak } \\
\text { firing (bits/s) }\end{array}$ & $254.9 \pm 54.37$ & $<0.0001$ & {$[164.9]$} & \\
\hline ISI distance before M & $0.716 \pm 0.301$ & 0.223 & {$[50.18]$} & \\
\hline $\begin{array}{l}\text { ISI distance at peak } \\
\text { firing }\end{array}$ & $0.558 \pm 0.244$ & & {$[164.9]$} & \\
\hline Peak ISI distance & $3.166 \pm 1.889$ & & $79.92 \pm 13.88$ & \\
\hline
\end{tabular}

The two-tailed paired $t$ test was used when the data were normally distributed. Otherwise, the Wilcoxon's signedrank test was used and is indicated by (W). Square brackets indicate times chosen as measuring points of the parameters. M, Muscimol; $R_{\mathrm{AV}}$, information capacity by averaging; $R_{\mathrm{C} 0}$, information capacity by coherence function.

established methods. In particular, ISI distance is inherently self-scaling and therefore insensitive to changes in timescale and firing rate (Kreuz et al., 2007).

Statistical inference. VassarStats (http://faculty.vassar.edu/lowry/ VassarStats.html) was used for all statistical tests. Correlated pairs of values (before and after changed condition) were tested by the twosample $t$ test for correlated samples when the data were normally distributed and by the Wilcoxon's signed-rank test when not normally distributed. Statistical significance in the figures is indicated by asterisks: ${ }^{\star} p<0.05,{ }^{* *} p<0.01,{ }^{* *} p<0.001$.

Data sufficiency. Because of the relatively limited amount of data available for each estimate of information capacity, we checked for sufficient data by reanalyzing all the muscimol data using only the initial $90 \%$ of the data for each point. The mean differences between the 100 and $90 \%$ values were $2.97 \%$ for information capacity by averaging, $1.64 \%$ for information capacity by coherence, and $6.12 \%$ for entropy rate.

\section{Results}

\section{Muscimol increased firing rate and information capacity}

Ten VS-3 neurons were treated by addition of $0.5 \mathrm{ml}$ of muscimol (100 $\mu \mathrm{M}$ solution), an agonist of $\mathrm{GABA}_{\mathrm{A}}$ receptors. The agent was applied after the neuron had received $60 \mathrm{~s}$ of random stimulation, which then continued for a total of $>300 \mathrm{~s}$ (Fig. 2, Table 1). Initialization of random stimulation causes rapid firing in VS-3 neurons, which adapts to a steady firing level with a time constant of $\sim 18 \mathrm{~s}$ (Höger and French, 2005). Delaying the application of modulating agents by $60 \mathrm{~s}$ allowed separation of this adaptation from the effects of modulation (Pfeiffer et al., 2009). Muscimol caused an increase in mean firing rate from 14.5 to $33.1 \mathrm{AP} / \mathrm{s}$, which was statistically significant (Table 1) and similar to previ- ous results in this preparation (Pfeiffer et al., 2009). The peak increase in firing occurred just over $100 \mathrm{~s}$ after the agent was added.

Information capacity calculated by both averaging, $R_{\mathrm{AV}}$, and by coherence function, $R_{\mathrm{CO}}$, increased after muscimol application but with clear differences in behavior. Values of $R_{\mathrm{AV}}$ were $\sim 10$ times greater than $R_{\mathrm{CO}}$. They were not significantly higher than before muscimol treatment when the neurons reached peak firing rate and reached their maximum values much later than any other changes in measured parameters, nearly $200 \mathrm{~s}$ after muscimol addition. In contrast, $R_{\mathrm{CO}}$ increased significantly by the peak firing time and reached a maximum $<30$ s later. Both information capacity measurements were reduced from their initial values immediately after muscimol application and for part of the time that firing rate was increasing.

\section{Muscimol increased entropy rate but not action potential timing reliability}

Entropy rate, or total information rate, increased rapidly after muscimol addition, reached a peak significantly before peak firing, and was still significantly elevated at peak firing (Fig. 2, Table 1). Maximum entropy rate is a nonlinear function of firing rate but increases approximately linearly with firing rate in this range of firing rates and sample intervals (French et al., 2003). Therefore, maximum entropy rate always reached a peak at the peak firing rate.

Interspike interval distance, a normalized measure of action potential timing jitter, or its inverse, reliability, always increased dramatically in the first few minutes after muscimol addition but then fell again and was not significantly different by the time of peak firing (Fig. 2).

\section{Increased firing rate alone mimicked some effects of muscimol}

In 10 different VS-3 neurons, the action potential firing rate was increased by current injection using the voltage-clamp controlled current-clamp method. This technique provides control of slow fluctuations in membrane potential, including the mean, but allows rapid membrane potential fluctuations to proceed normally. Mean values of the various parameters were obtained from 50 s periods before and after the depolarization (Fig. 3, Table 2). The mean depolarization of $20 \mathrm{mV}$ increased the mean firing rate from $\sim 13$ to $\sim 23 \mathrm{AP} / \mathrm{s}$. Information capacity estimated from the coherence function and entropy rate both increased significantly at the higher firing rate. However, information capacity estimated by averaging did not change significantly. ISI distance again increased strongly during the change in firing rate but then fell again and was significantly lower at the higher firing rate.

\section{Discussion}

$\mathrm{GABA}_{\mathrm{A}}$ receptor agonists produce a triphasic response in VS-3 neurons, consisting of an initial brief excitation, then a rapid inhibition, followed by long-lasting excitation (Pfeiffer et al., 2009). This finding was repeated here. We also confirmed that information capacity, measured by two different methods, increased significantly during the excitation period (21\% by averaging, $26 \%$ by coherence). However, the absolute values of information capacity given by the two methods were very different $(\sim 10$ times greater by averaging) and the time courses of their change after muscimol were also different, with $R_{\mathrm{CO}}$ reaching a maximum earlier, close to the peak in action potential firing, than seen in $R_{\mathrm{AV}}$, which was not significantly elevated at the time of peak firing. 


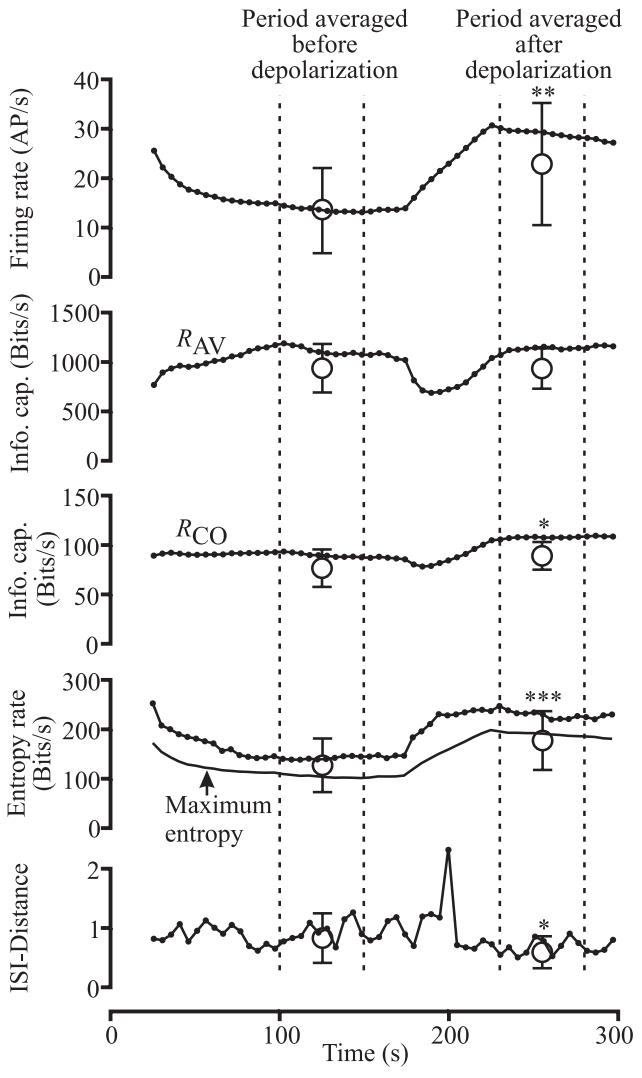

Figure 3. Effects of changing the firing rate of VS-3 neurons by voltage-clamp controlled current clamp. Membrane potential was held at the resting potential $(-73.1 \pm 6.3 \mathrm{mV})$ for 200 s and then depolarized by $\sim 20 \mathrm{mV}$ to $-54.2 \pm 6.5 \mathrm{mV}$ and held at the new potential for 100 s. Data from one neuron (filled circles and lines joining them) are shown as illustration, with open circles and vertical bars showing the mean \pm SD values from 10 different neurons superimposed. Mean values of firing rate, information capacity (by averaging and by coherence), entropy, and ISI distance were obtained from $50 \mathrm{~s}$ periods before and after the depolarization (100 - 150 and $230-280$ s indicated by dashed vertical lines). Parameters were estimated by identical methods to those used for the muscimol experiments (Fig. 2). $R_{\mathrm{Av}}$, Information capacity by averaging; $R_{\mathrm{CO}}$, information capacity by coherence function.

Table 2. Effects of raising the action potential firing rate by current injection on information capacity, entropy rate, and interspike interval distance in 10 different VS-3 neurons

\begin{tabular}{llll}
\hline Parameter & $\begin{array}{l}\text { Resting } \\
\text { Mean } \pm \text { SD }\end{array}$ & $\begin{array}{l}\text { Depolarized } \\
\text { Mean } \pm \text { SD }\end{array}$ & $p$ \\
\hline Firing rate (AP/s) & $13.47 \pm 8.69$ & $22.96 \pm 12.46$ & 0.001 \\
$R_{\text {AV }}(\mathrm{bits} / \mathrm{s})$ & $937.6 \pm 244.8$ & $934.4 \pm 203.3$ & 0.953 \\
$R_{\text {CO }}(\mathrm{bits} / \mathrm{s})$ & $76.59 \pm 19.00$ & $89.27 \pm 13.93$ & 0.018 \\
Entropy (bits/s) & $127.3 \pm 54.2$ & $177.5 \pm 59.6$ & 0.0002 \\
ISI distance & $0.8308 \pm 0.4182$ & $0.5914 \pm 0.2676$ & $0.025(\mathrm{~W})$
\end{tabular}

The two-tailed paired $t$ test was used for normally distributed data. Otherwise, the Wilcoxon's signed-rank test (W) was used. $R_{\mathrm{AV}}$, Information capacity by averaging; $R_{\mathrm{CO}}$, information capacity by coherence function.

Both methods of calculating information capacity integrated estimates of SNR over frequency. Because a constant frequency range was used for all experiments, the information capacity values reported here were directly proportional to average SNR values in all cases. Therefore, SNR values are not shown in the figures to avoid repetition. They reached $\sim 5.0$ by averaging and $\sim 0.15$ by coherence function.

\section{Does inherent membrane noise limit information capacity?}

The large divergence in absolute values of information capacity by the averaging and coherence methods reflects major differ- ences in their analytical bases. Although both attempt to estimate SNR, the averaging approach measures variability or noise in the response without reference to the input signal, whereas coherence function measures the linear correlation between input and output, so that measuring information capacity by this method assumes that deviations from a linear, noise-free response are attributable to noise added by the system, which may not be true.

The relatively high information capacity values given by averaging indicate that inherent noise or variability in VS-3 neurons is actually low and does not significantly limit the information capacity of mechanotransduction.

Reliability of action potential timing offers an alternative view of neuronal noise, and a range of analytical techniques have been used to measure timing variability, or jitter (for review, see Billimoria et al., 2006; Kreuz et al., 2007). The ISI distance method used here is a normalized estimate of jitter, with a value of 0 for perfect reliability and increasingly positive values as action potential timing becomes more unpredictable. ISI distance increased dramatically when muscimol was added but soon fell again and was not significantly different at the time of maximum firing. This agrees with the averaging data and supports the ideas that inherent noise is low and that muscimol application, with its resultant increase in firing rate, did not decrease noise.

Information capacity estimated by averaging did increase significantly much later in the experiments. The reasons for this are not clear, but Pfeiffer et al. (2009) also found a delayed component of excitation that was not dependent on depolarization. This delayed effect of muscimol may also cause an increase in SNR.

\section{Inherent noise increased transiently after muscimol application}

ISI distance increased dramatically when muscimol was added but soon fell again and was not significantly different at the time of maximum firing. Information capacity, and hence SNR, also decreased strongly during the time that the firing rate was increasing (e.g., between 60 and $120 \mathrm{~s}$ in Fig. 2). Of course, it is impossible to increase the mean rate of firing without changes in the patterns of action potentials during each random stimulus presentation, but the low SNR, high ISI distance and rapid rise in entropy rate all indicate an increase in inherent noise during the transition from low to high firing rate. One explanation for this could be noise caused by the change from low to high ion channel open probability caused by muscimol. Membrane noise and current can be used to estimate open channel probability (Defelice, 1981; Traynelis and Jaramillo, 1998), with maximum membrane noise occurring at $50 \%$ open probability.

Similar arguments have been made for changing membrane noise by neurotransmitter activated ion channels in crab mechanoreceptors (Billimoria et al., 2006). Our findings contrast with the crab mechanoreceptor data because we found that noise decreased as firing rate increased, whereas Billimoria et al. (2006), using two different neurotransmitters, found the opposite effect. If the change in noise is primarily linked to open probability of neurotransmitter-activated channels, this difference may reflect differing abilities of the chemicals to exceed $50 \%$ channel opening. This might also explain why $R_{\mathrm{AV}}$ reached a maximum late in the present experiments.

\section{Does entropy rate limit information capacity?}

Entropy rate estimates how much information is present in the action potential signal, without considering how much of the action potential timing represents encoded mechanical signal. In 
contrast, maximum entropy rate is the amount of information that could be encoded in an action potential train at a given firing rate (Rieke et al., 1997). A perfectly regular action potential train would have a very low entropy rate, regardless of firing rate or maximum entropy rate.

The data compression method of estimating entropy has the advantage of being independent of any assumptions about the mechanism of information coding. However, it often gives higher values than the maximum entropy rate. Possible reasons for this include a failure to achieve maximal possible compression and the use of Stirling's approximation in the derivation of maximum entropy (French et al., 2003).

Entropy rate and maximum entropy rate had similar absolute values to the coherence-based information capacity, suggesting that information capacity is limited by available entropy in the action potential signal. However, entropy rate peaked earlier during muscimol treatment than the coherence-based measure and earlier than the firing rate (and hence the maximum entropy rate), indicating that another source of entropy was present during that part of the experiment. This seems likely to represent the noise added by ion channel opening, as described above.

Depolarization of VS-3 neurons by current injection increased the action potential firing rate but did not reduce inherent neuronal noise, as measured by the averaging information capacity. ISI distance did decrease at the higher firing rate, although only at $p=0.025$ (Fig. 3, Table 2). However, entropy rate and coherence-based information capacity both increased significantly with firing rate, supporting the idea that increased firing raises the maximum entropy rate available, and this is used by the neural encoder to encode information about the mechanical stimulus.

\section{Conclusions}

Previous investigations have mainly emphasized the excitation or inhibition produced by neuromodulation, but other effects, such as changing dynamic sensitivity or inherent noise, may also be important. Our data indicate that GABAergic excitation of spider mechanoreceptors increases the ability of the neurons to transmit mechanosensory information by increasing the entropy level that can be encoded in the action potential train. This effect seems much greater than changes in membrane noise level. Given the widespread occurrence of neuromodulation and close similarities between neuromodulation and synaptic integration, it seems important to consider possible roles of information carrying capacity, as well as neuronal noise levels, when exploring neurotransmitter functions.

\section{References}

Aldworth ZN, Miller JP, Gedeon T, Cummins GI, Dimitrov AG (2005) Dejittered spike-conditioned stimulus waveforms yield improved estimates of neuronal feature selectivity and spike-timing precision of sensory interneurons. J Neurosci 25:5323-5332.

Bendat JS, Piersol AG (1980) Engineering applications of correlation and spectral analysis. New York: Wiley.

Billimoria CP, DiCaprio RA, Birmingham JT, Abbott LF, Marder E (2006) Neuromodulation of spike-timing precision in sensory neurons. J Neurosci 26:5910-5919.

Birmingham JT, Billimoria CP, DeKlotz TR, Stewart RA, Marder E (2003) Differential and history-dependent modulation of a stretch receptor in the stomatogastric system of the crab, Cancer borealis. J Neurophysiol 90:3608-3616

Borst A, Theunissen FE (1999) Information theory and neural coding. Nat Neurosci 2:947-957.

Carlton SM (2001) Peripheral excitatory amino acids. Curr Opin Pharmacol 1:52-56.

Cooley JW, Tukey JW (1965) An algorithm for the machine calculation of complex Fourier series. Math Comput 19:297-301.

Defelice LJ (1981) Introduction to membrane noise. New York: Plenum.

French AS, Holden AV (1971) Alias-free sampling of neuronal spike trains. Kybernetik 8:165-171.

French AS, Höger U, Sekizawa S-i, Torkkeli PH (2001) Frequency response functions and information capacities of paired spider mechanoreceptor neurons. Biol Cybern 85:293-300.

French AS, Höger U, Sekizawa S-i, Torkkeli PH (2003) A context-free data compression approach to measuring information transmission by action potentials. Biosystems 69:55-61.

Höger U, French AS (2005) Slow adaptation in spider mechanoreceptor neurons. J Comp Physiol A Neuroethol Sens Neural Behav Physiol 191:403-411.

Jiménez-Montaño MA, Penagos H, Hernández Torres A, Diez-Martínez O (2000) Measures of complexity in neural spike-trains of the slowly adapting stretch receptor organs. Biosystems 58:117-124.

Juusola M, French AS (1997) The efficiency of sensory information coding by mechanoreceptor neurons. Neuron 18:959-968.

Juusola M, Seyfarth EA, French AS (1994) Sodium-dependent receptor current in a new mechanoreceptor preparation. J Neurophysiol 72:3026-3028.

Kreuz T, Haas JS, Morelli A, Abarbanel HD, Politi A (2007) Measuring spike train synchrony. J Neurosci Methods 165:151-161.

Loewenstein WR (1956) Modulation of cutaneous mechanoreceptors by sympathetic stimulation. J Physiol 132:40-60.

Matheson T (1997) Octopamine modulates the responses and presynaptic inhibition of proprioceptive sensory neurones in the locust Schistocerca gregaria. J Exp Biol 200:1317-1325.

Page AJ, Blackshaw LA (1999) GABA $_{B}$ receptors inhibit mechanosensitivity of primary afferent endings. J Neurosci 19:8597-8602.

Pfeiffer K, Panek I, Höger U, French AS, Torkkeli PH (2009) Random stimulation of spider mechanosensory neurons reveals long-lasting excitation by GABA and muscimol. J Neurophysiol 101:54-66.

Ramirez J-M, Büschges A, Kittmann R (1993) Octopaminergic modulation of the femoral chordotonal organ in the stick insect. J Comp Physiol A Neuroethol Sens Neural Behav Physiol 173:209-219.

Rieke F, Warland DD, de Ruyter van Steveninck R, Bialek W (1997) Spikes. Exploring the neural code. Cambridge, MA: MIT.

Rokem A, Watzl S, Gollisch T, Stemmler M, Herz AV, Samengo I (2006) Spike-timing precision underlies the coding efficiency of auditory receptor neurons. J Neurophysiol 95:2541-2552.

Rudomin P, Schmidt RF (1999) Presynaptic inhibition in the vertebrate spinal cord revisited. Exp Brain Res 129:1-37.

Seyfarth EA, French AS (1994) Intracellular characterization of identified sensory cells in a new spider mechanoreceptor preparation. J Neurophysiol 71:1422-1427.

Shannon CE, Weaver W (1949) The mathematical theory of communication. Chicago: University of Illinois.

Sutor B, Grimm C, Polder HR (2003) Voltage-clamp-controlled currentclamp recordings from neurons: an electrophysiological technique enabling the detection of fast potential changes at preset holding potentials. Pflugers Arch 446:133-141.

Torkkeli PH, Panek I (2002) Neuromodulation of arthropod mechanosensory neurons. Microsc Res Tech 58:299-311.

Traynelis SF, Jaramillo F (1998) Getting the most out of noise in the central nervous system. Trends Neurosci 21:137-145.

Widmer A, Höger U, Meisner S, French AS, Torkkeli PH (2005) Spider peripheral mechanosensory neurons are directly innervated and modulated by octopaminergic efferents. J Neurosci 25:1588-1598. 